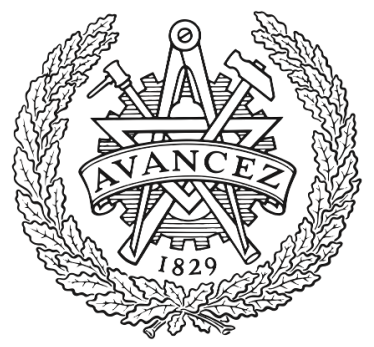

CHALMERS

UNIVERSITY OF TECHNOLOGY

\title{
Elimination/Mitigation of Output Voltage Harmonics for Multilevel Converters Operated at Fundamental Switching Frequency using Matlab's
}

Downloaded from: https://research.chalmers.se, 2023-04-26 01:05 UTC

Citation for the original published paper (version of record):

Kersten, A., Kuder, M., Singer, A. et al (2020). Elimination/Mitigation of Output Voltage Harmonics for Multilevel Converters Operated at

Fundamental Switching Frequency using Matlab's Genetic Algorithm Optimization. 2020 22nd

European Conference on Power Electronics and Applications, EPE 2020 ECCE Europe: 1-12.

http://dx.doi.org/10.23919/EPE20ECCEEurope43536.2020.9215791

N.B. When citing this work, cite the original published paper. 


\title{
Elimination/Mitigation of Output Voltage Harmonics for Multilevel Converters Operated at Fundamental Switching Frequency using Matlab's Genetic Algorithm Optimization
}

\author{
Anton Kersten ${ }^{1}$, Manuel Kuder ${ }^{2}$, Arthur Singer ${ }^{3}$, Weiji Han ${ }^{1}$, \\ Torbjörn Thiringer ${ }^{1}$, Thomas Weyh ${ }^{2}$, and Richard Eckerle ${ }^{2}$ \\ ${ }^{1}$ Chalmers University of Technology, Gothenburg, Sweden \\ Email: kersten@chalmers.se \\ ${ }^{2}$ University of the German Federal Armed Forces, Munich, Germany \\ Email: manuel.kuder@unibw.de \\ ${ }^{3}$ STABL Energy (m-Bee GmbH), Munich, Germany \\ Email: arthur.singer@stabl.com
}

September 2020

\begin{abstract}
Keywords
$\ll$ Battery $\gg, \ll$ Cascaded $\gg, \ll$ Efficiency $\gg, \ll$ Electric vehicle $\gg, ~ \ll$ Energy storage $\gg, \ll$ H-bridge $\gg$, $\ll$ IGBT $\gg, \ll$ MOSFET $\gg, \ll$ Multilevel converter $\gg, \ll$ Multilevel system $\gg, \ll$ Switching losses $\gg$.
\end{abstract}

\begin{abstract}
This paper deals with the optimization of the output voltage waveform of a multilevel converter operated with fundamental frequency switching. For a high number of output voltage levels, nearest-level control is typically used, whereas an optimized waveform can be presumably used to eliminate a selection of low order harmonics. A nonlinear optimization problem for any kind of multilevel inverter, operating in a single or three-phase arrangement, is formulated. It is shown that the set of nonlinear equations, defining this optimization problem, cannot be numerically solved, if the number of output voltage levels is higher than nine. Thus, an optimization algorithm, e.g., Matlab's genetic algorithm, should be used instead. Based on the concept of the weighted THD, it is shown that an optimized waveform has no effect on the output current's quality of a single phase multilevel converter. However, considering an ungrounded three-phase system, the content of the to be eliminated harmonic components is shifted towards the triplen harmonics and, consequently, the expected current quality, based on the WTHD, can be significantly improved.
\end{abstract}

\section{Introduction}

The classical modular multilevel converter topology, as presented in [1], is typically used for power system applications as HVDC. However, different variants of multilevel converters are not just gaining in interest in the field of large electric drives [2,3], these are becoming also popular for low voltage applications such as transportation electrification [4], renewable energy sources [5, 6] and energy storages $[7,8,9]$. Their main advantages for low voltage and variable speed drive applications can be summarized as follows: fault tolerant operation [10], increased partial load efficiency through the usage of low voltage MOSFETs in comparison to IGBTs $[11,12,13,14]$ and, especially, reduced electromagnetic emissions [15, 16, 17].

Different output voltage modulation techniques can be found in [18]. Nearest-level control (NLC) [19] is a commonly used fundamental frequency switching technique to synthesize the desired sinusoidal output voltage. According to [18], the pulse positions to activate each sub-module in forward or reverse 
direction are crucial for the content of low order harmonics. A proper placement of the pulse positions can be used to eliminate a selection of low order harmonics. To find the proper pulse positions, a set of nonlinear equations must be solved. Solving these numerically for the seven-level inverter can already require an extensive computational effort [20]. In [20,21, 22], an approach using resultants and symmetrical polynomials is presented, which is quite cumbersome. On the contrary, an optimization approach can be used instead to find a solution apart from the global optimum, which requires less computational effort. In [23], a genetic algorithm approach is chosen, whereas the number of output levels is just limited to seven. Furthermore, the presented approaches in $[18,20,21,22,23]$ only refer to converters operated in a three-phase arrangement and only up to eleven output voltage levels are considered.

Therefore, this paper presents a generalized optimization problem for the output voltage waveform of a multilevel converter with the goal to eliminate/mitigate a selection of odd harmonics, including and excluding the triplen harmonics. This optimization problem, consisting of a set of nonlinear equation, is linearized and numerically solved for up to nine output voltage levels. For a larger number of output voltage levels, Matlab's Genetic Algortihm (GA) approach is used. To assess the effectiveness of the optimized waveform in comparison to NLC, the concept of the Weighted Total Harmonic Distoriton (WTHD) is used.

\section{Multilevel Converter Topology and Output Voltage Waveform}

The described approaches in this paper can be applied to different kinds of multilevel inverters, for example the ones described in $[1,12,24]$, with any number of output levels $L$. Within the frame of this paper's analysis, the example of a grid-tied Cascaded H-Bridge (CHB) converter with integrated battery storages and an arbitrary number of output levels $L$, as can be seen in Fig. 1, is considered. Due to the utilization of small battery modules, the individual DC-link voltages do not fluctuate as much when using only capacitors. This type of converter could be used for different applications as for example a battery storage system or a vehicle inverter, which can be connected to the three-phase mains, working as a charger. Each H-bridge has three valid switching states, achieving three output voltages levels according

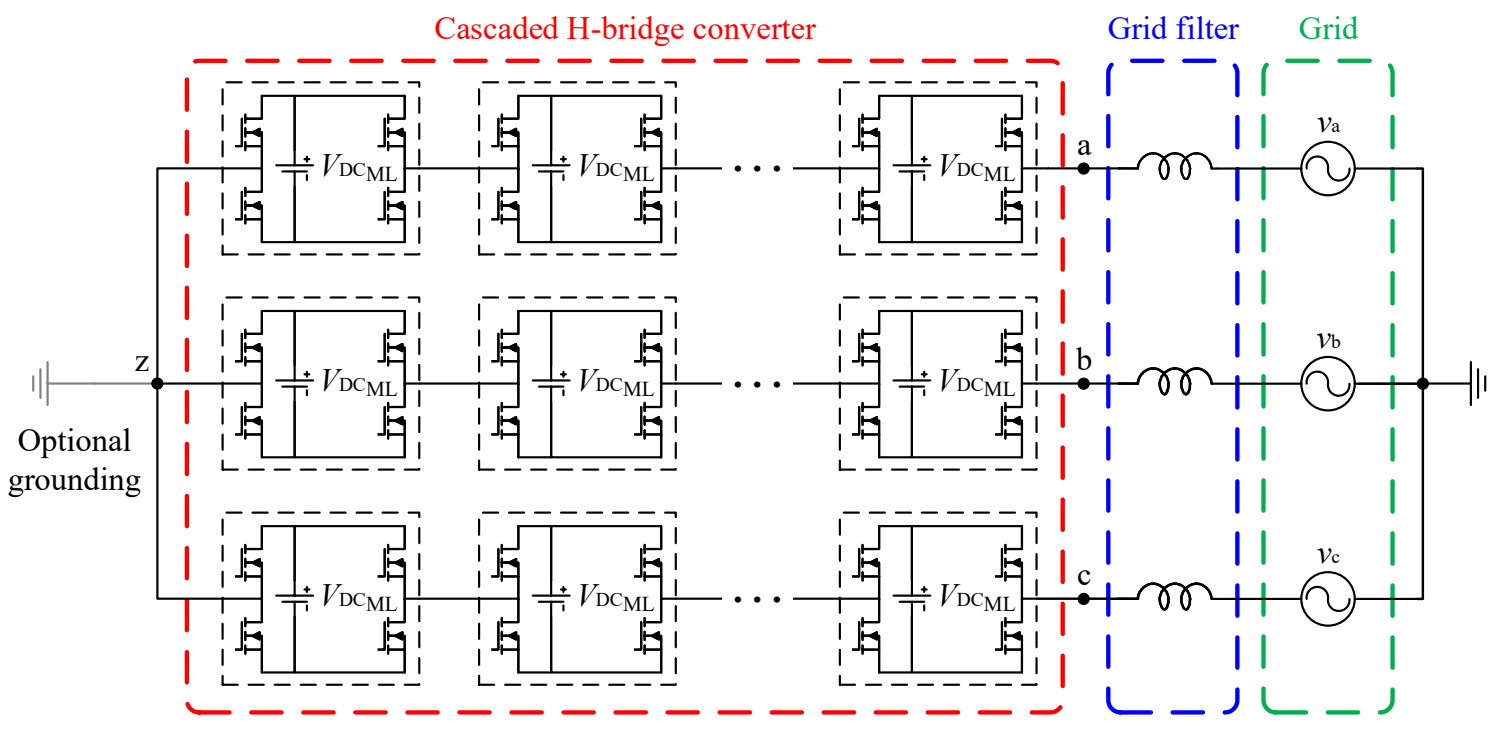

Fig. 1: Grid-connected cascaded H-bridge (CHB) converter.

to

$$
v_{\mathrm{HB} j}(t)=\left\{+V_{\mathrm{DC}_{\mathrm{ML}}} ;-V_{\mathrm{DC}} ; 0\right\} .
$$


The phase voltage of each strand can be calculated by the sum of the output voltages of the individual H-bridges, with $m=\frac{L-1}{2}$ being the number of modules per phase, as

$$
v_{\text {phase }}(t)=v_{\mathrm{az}}(t)=\sum_{j=1}^{m=\frac{L-1}{2}} v_{\mathrm{HB} j}(t)
$$

Using fundamental frequency switching, each H-bridge is switched only once per half period. Thus, the switching-time instants of each H-bridge can be expressed by a vector of switching angles $\alpha$ according to

$$
\alpha=\left[\begin{array}{c}
\alpha_{1} \\
: \\
\alpha_{\frac{L-1}{2}}
\end{array}\right] \quad \text { with } \quad v_{\mathrm{HB} j}\left(\alpha_{j}\right)=\left\{\begin{array}{cl}
+V_{\mathrm{DC}} ; & \text { if } \alpha_{j} \leq \omega t \leq \pi-\alpha_{j} \\
-V_{\mathrm{DC}} ; & \text { if } \pi+\alpha_{j} \leq \omega t \leq 2 \pi-\alpha_{j} \\
0 ; & \text { else }
\end{array}\right.
$$

In this manner, a staircase-shaped voltage waveform, as shown in Fig. 2(a), can be built up. The corresponding current, drawn from one battery module, is depicted in Fig. 2(b). According to [18], the

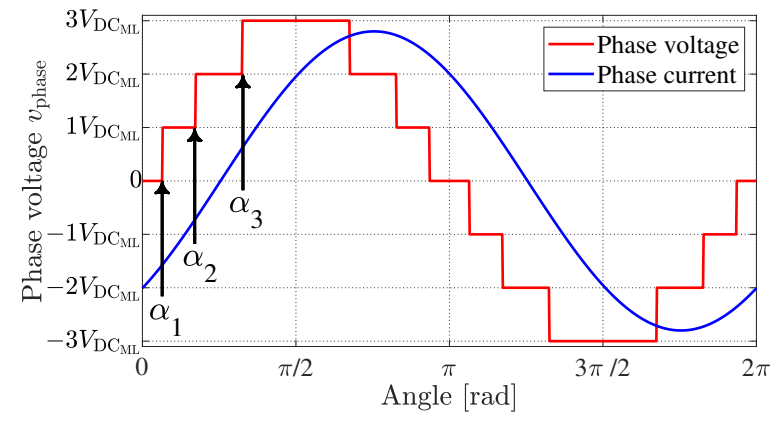

(a)

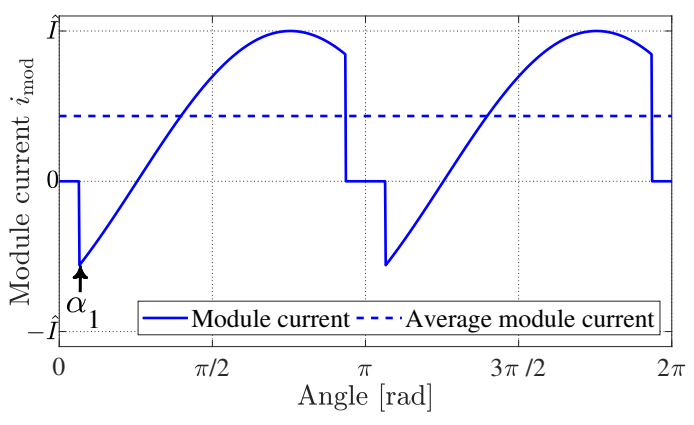

(b)

Fig. 2: (a) Phase voltage and phase current waveforms of the cascaded H-bridge converter. (b) Drawn battery current of an individual H-bridge module.

fundamental component and the harmonic components of the stair-case shaped phase voltage waveform can be expressed as

$$
V_{\mathrm{az}, h}=\frac{8 V_{\mathrm{DC}}}{(L-1) h \pi}\left(\cos \left(h \alpha_{1}\right)+\ldots+\cos \left(h \alpha_{\frac{L-1}{2}}\right)\right) \quad \text { with } \quad h=\{1,3,5, \ldots\} .
$$

The DC-link voltage $V_{\mathrm{DC}}$ corresponds to the sum of the individual DC-link voltages according to

$$
V_{\mathrm{DC}}=m V_{\mathrm{DC}}
$$

\section{Concept of Weighted THD (WTHD)}

The Weighted Total Harmonic Distortion (WTHD), as described in [18], can be used to assess and compare the probable current quality of different voltage waveforms, when applied to a lossless inductance. To explain the concept of the WTHD, it is reasonable to start from the voltage THD expression, which can be described as

$$
T H D_{\mathrm{v}}=\sqrt{\left(\frac{V_{\mathrm{rms}}}{V_{1, \mathrm{rms}}}\right)^{2}-1} .
$$

Without a DC component, the voltage THD expression becomes

$$
T H D_{\mathrm{v}}=\sqrt{\sum_{h=2}^{\infty}\left(\frac{V_{h}}{V_{1}}\right)^{2}} .
$$


Similar as in (7), the current THD can be expressed as

$$
T H D_{\mathrm{i}}=\sqrt{\sum_{h=2}^{\infty}\left(\frac{I_{h}}{I_{1}}\right)^{2}} .
$$

Considering a lossless inductive load, the current harmonics can be calculated with the help of the voltage harmonics according to

$$
I_{h} \approx \frac{V_{h}}{h \omega_{1} L} \quad \text { with } \quad h=\{2,3,4 . .\} \quad .
$$

Inserting (9) in the current THD expression given in (9), the weighted THD as a function of the voltage harmonics can be obtained according to

$$
W T H D=\frac{1}{V_{1}} \sqrt{\sum_{h=2}^{\infty}\left(\frac{V_{h}}{h}\right)^{2}} .
$$

Due to the reason that the even voltage harmonics are zero, the WTHD for a single phase system can be described as

$$
W T H D_{1, \mathrm{Ph}}=\frac{1}{V_{1}} \sqrt{\sum_{h=3,5,7 \ldots}^{\infty}\left(\frac{V_{h}}{h}\right)^{2}} \text { with } h=h_{1, \mathrm{Ph}}=\{3,5,7 \ldots\}=\sum_{i=1}^{\infty} i \cdot 2+1 .
$$

Further, the triplen voltage harmonics in a three-phase system with only one grounding, point, as for example shown in Fig. 1, cancel each other out among the phases and, thus, these do not cause any currents to flow. Thus, the WTHD, excluding the triplen harmonics can be expressed as

$$
W \operatorname{THD}_{3, \mathrm{Ph}}=\frac{1}{V_{1}} \sqrt{\sum_{h=5,7,11 \ldots}^{\infty}\left(\frac{V_{h}}{h}\right)^{2}} \text { with } h=h_{3, \mathrm{Ph}}=\{5,7,11 \ldots\}=\sum_{i=1}^{\infty} i \cdot 6 \pm 1 .
$$

\section{Nearest-Level Control}

A simple approach to synthesize the desired sinusoidal output voltage waveform is Nearest-Level Control (NLC), as described in [18]. The fundamental component can be approximated with the help of the modulation index $M$ according to

$$
\hat{V}_{\mathrm{az}, 1} \approx V_{\mathrm{DC}} M \quad \text { with } \quad V_{\mathrm{DC}}^{\prime}=\frac{2 V_{\mathrm{DC}}}{L-1} .
$$

If the modulation index is low, not all voltage levels are needed. With respect to $\alpha$, the number of needed insertion angles can be calculated as

$$
k=\left\lceil\frac{M(L-1)}{2}\right\rceil,
$$

where the operator \lceil\rceil indicates to round up the result to the nearest integer value. The value of the insertion angles can be calculated according to

$$
(j-0.5) V_{\mathrm{DC}}^{\prime}=\frac{(2 j-1) V_{\mathrm{DC}}}{L-1}=V_{\mathrm{DC}} M \sin \left(\alpha_{n}\right) \quad \rightarrow \quad \alpha_{j}=\arcsin \left(\frac{2 j-1}{(L-1) M}\right)
$$


and the insertion angle vector $\alpha$ becomes

$$
\alpha=\left[\begin{array}{c}
\alpha_{1}=\arcsin \left(\frac{1}{(L-1) M}\right) \\
\vdots \\
\alpha_{k}=\arcsin \left(\frac{2 k-1}{(L-1) M}\right) \\
\alpha_{k+1}=\frac{\pi}{2} \\
\vdots \\
\alpha_{\frac{L-1}{2}}=\frac{\pi}{2}
\end{array}\right] .
$$

\section{Fundamental Selective Harmonic Elimination/Mitigation}

Another method to synthesize the desired sinusoidal output voltage waveform is Fundamental Selective Harmonic Elimination. As described in [18], through the adjustment of the insertion angles $\alpha$, an $L$-level converter can control up to $\frac{L-1}{2}$ voltage components, including the fundamental component. Hence, a selection of low order harmonics can be controlled to be zero. For example, to find the proper values of $\alpha$ for a three-phase converter (excluding even and triplen harmonics) operating at a modulation index $M$ close to unity, the following set of nonlinear equations must be solved:

$$
\begin{aligned}
V_{\mathrm{az}, 1} & =\frac{8 V_{\mathrm{DC}}}{(L-1) 1 \pi}\left(\cos \left(1 \alpha_{1}\right)+\ldots+\cos \left(1 \alpha_{\frac{L-1}{2}}\right)\right)=M V_{\mathrm{DC}} \\
V_{\mathrm{az}, 5} & =\frac{8 V_{\mathrm{DC}}}{(L-1) 5 \pi}\left(\cos \left(5 \alpha_{1}\right)+\ldots+\cos \left(5 \alpha_{\frac{L-1}{2}}\right)\right)=0 \\
V_{\mathrm{az}, 7} & =\frac{8 V_{\mathrm{DC}}}{(L-1) 7 \pi}\left(\cos \left(7 \alpha_{1}\right)+\ldots+\cos \left(7 \alpha_{\frac{L-1}{2}}\right)\right)=0 \\
\vdots & \\
V_{\mathrm{az}, h_{\max }} & =\frac{8 V_{\mathrm{DC}}}{(L-1) h_{\max } \pi}\left(\cos \left(h_{\max } \alpha_{1}\right)+\ldots+\cos \left(h_{\max } \alpha_{\frac{L-1}{2}}\right)\right)=0
\end{aligned}
$$

The nonlinear equation system in (17) can be numerically solved. However, due to the nonlinear trigonometric functions $\cos \left(h \alpha_{n}\right)$, the computational effort is quite high. Thus, the expressions for the harmonics can be linearized using the following term

$$
\cos \left(h \alpha_{n}\right)=\sum_{j=0}^{\left\lfloor\frac{h}{2}\right\rfloor}(-1)^{j}\left(\begin{array}{c}
h \\
2 j
\end{array}\right) \sin ^{2 j}\left(\alpha_{n}\right) \cos ^{h-2 j}\left(\alpha_{n}\right) \quad \text { with } \quad n=\left\{1,2,3, \ldots, \frac{L-1}{2}\right\}
$$

with the binomial coefficient according to

$$
\left(\begin{array}{c}
h \\
2 j
\end{array}\right)=\frac{h !}{(2 j) !(h-2 j) !} .
$$

For example, for $h=3$, (18) becomes

$$
\begin{aligned}
\cos \left(3 \alpha_{n}\right) & =\sum_{j=0}^{\left\lfloor\frac{3}{2}\right\rfloor}(-1)^{j}\left(\begin{array}{c}
3 \\
2 j
\end{array}\right) \sin ^{2 j}\left(\alpha_{n}\right) \cos ^{3-2 j}\left(\alpha_{n}\right) \\
\cos \left(3 \alpha_{n}\right) & =\left.\left[\cos ^{3}\left(\alpha_{n}\right)\right]\right|_{j=0}+\left.\left[-3 \sin ^{2}\left(\alpha_{n}\right) \cos \left(\alpha_{n}\right)\right]\right|_{j=1}
\end{aligned}
$$

With the help of the trigonometric expression

$$
\sin ^{2}\left(\alpha_{n}\right)+\cos ^{2}\left(\alpha_{n}\right)=1 \quad \rightarrow \quad \sin ^{2}\left(\alpha_{n}\right)=1-\cos ^{2}\left(\alpha_{n}\right) \quad,
$$


(20) can be simplified and, thus, becomes

$$
\cos \left(3 \alpha_{n}\right)=4 \cos ^{3}\left(\alpha_{n}\right)-3 \cos \left(\alpha_{n}\right)
$$

Calculating (18), for a series of $h$, the following recursive relation can be obtained

$$
\begin{aligned}
\cos \left(0 \alpha_{n}\right) & =1 \\
\cos \left(1 \alpha_{n}\right) & =\cos \left(\alpha_{n}\right) \\
\cos \left(2 \alpha_{n}\right) & =2 \cos ^{2}\left(\alpha_{n}\right)-1 \\
\cos \left(3 \alpha_{n}\right) & =4 \cos ^{3}\left(\alpha_{n}\right)-3 \cos \left(\alpha_{n}\right) \\
\cos \left(4 \alpha_{n}\right) & =8 \cos ^{4}\left(\alpha_{n}\right)-8 \cos ^{2}\left(\alpha_{n}\right)+1 \\
\vdots & \\
\cos \left((j+1) \alpha_{n}\right) & =2 \cos \left(\alpha_{n}\right) \cos \left((j) \alpha_{n}\right)-\cos \left((j-1) \alpha_{n}\right)
\end{aligned}
$$

which is referred to as Chebyshev polynomials. In a three-phase arrangement, without the optional grounding as shown in Fig. 1, it is preferred to select the harmonics to be eliminated among a series of odd harmonics, excluding the triplen harmonics, according to

$$
H_{3, \text { phase }}=5,7,11,13,17,19,23,25,29,31 \ldots=\sum_{i=1}^{\infty} i \cdot 6 \pm 1
$$

whereas in a single-phase arrangement a selection among all odd harmonics should be made according to

$$
H_{1, \text { phase }}=3,5,7,9,11,13,15,17,19,21,23 \ldots=\sum_{i=1}^{\infty} i \cdot 2+1
$$

Listing 1 in the Appendix shows a numerical approach to solve the set of linearized equations for a certain modulation index $M$. As shown for a nine-level inverter, the calculation time is about $7 \mathrm{~min}$ and $18 \mathrm{~s}$. When increasing the number of levels beyond nine, the computational time increases significantly and, thus, the equation system cannot be numerically solved any longer. Therefore, the nonlinear equation system can be used to formulate the following optimization problem, considering a single or three-phase converter arrangement:

Single - Phase :

$$
\underset{\alpha}{\operatorname{minimize}} \quad \sum_{h=3,5,7 \ldots}^{L-2}(L+1-h) \frac{8 V_{\mathrm{DC}}}{(L-1) h \pi}\left|\sum_{n=1}^{\frac{L-1}{2}} \cos \left(h \alpha_{n}\right)\right|
$$

Three-Phase :

$$
\begin{aligned}
& \underset{\alpha}{\operatorname{minimize}} \\
& \sum_{h=5,7,11 \ldots}^{3 \frac{L-1}{2}-2}\left(\frac{3 L+3}{2}-h\right) \frac{8 V_{\mathrm{DC}}}{(L-1) h \pi}\left|\sum_{n=1}^{\frac{L-1}{2}} \cos \left(h \alpha_{n}\right)\right| \text { for odd } \frac{L-1}{2} \\
& \underset{\alpha}{\operatorname{minimize}} \\
& \sum_{h=5,7,11 \ldots}^{3 \frac{L-1}{2}-1}\left(\frac{3 L+3}{2}-h+(-1)^{\left\lceil\frac{h}{3}\right\rceil}\right) \frac{8 V_{\mathrm{DC}}}{(L-1) h \pi}\left|\sum_{n=1}^{\frac{L-1}{2}} \cos \left(h \alpha_{n}\right)\right| \quad \text { for even } \frac{L-1}{2}
\end{aligned}
$$

Constraints :

subject to

$$
\begin{aligned}
& V_{\mathrm{az}, 1}=\frac{8 V_{\mathrm{DC}}}{(L-1) \pi}\left(\cos \left(\alpha_{1}\right)+\ldots+\cos \left(\alpha_{\frac{L-1}{2}}\right)\right)=V_{\mathrm{DC}} M \\
& 0 \leq \alpha_{1} \leq \alpha_{2} \leq \ldots . \leq \alpha_{\frac{L-1}{2}} \leq \frac{\pi}{2}
\end{aligned}
$$


To solve the formulated problem in (26), Matlab's Genetic Algorithm (GA) can be used. Solving the optimization can be faster than a pure numerical approach, whereas the obtained solution might not equal the global optimum. Listing 2 in the Appendix shows the Matlab code for the optimization using the genetic algorithm.

\section{Optimized Voltage Waveform for a 17-Level Converter}

A multilevel converter with 17 output levels is considered to assess the effectiveness of the introduced optimization approaches in comparison to nearest-level control. For the optimization problem, a three-phase arrangement with and without the optional grounding, as show in Fig. 1 and described in (26), is considered. The insertion angles are optimized relative to the modulation index $M$ using Mat-

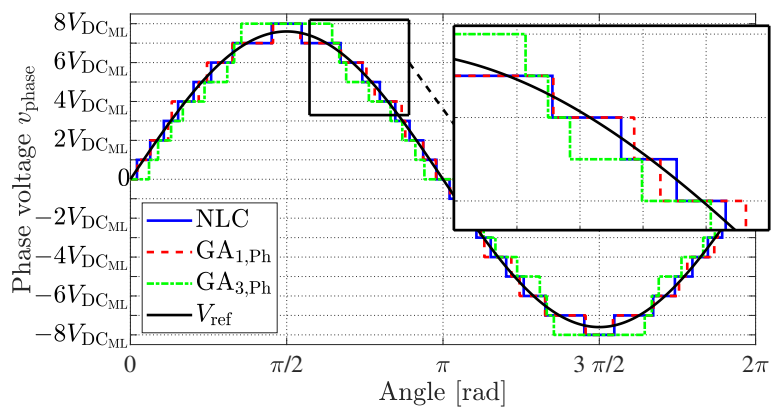

(a)

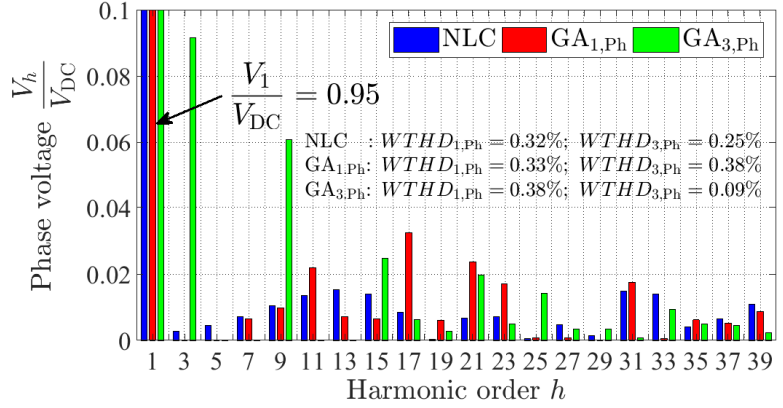

(b)

Fig. 3: (a) Synthesized reference voltage using NLC and optimized insertion angles and (b) corresponding harmonic components, including $W T H D_{1, \mathrm{Ph}}$ and $W T H D_{3, \mathrm{Ph}}$.

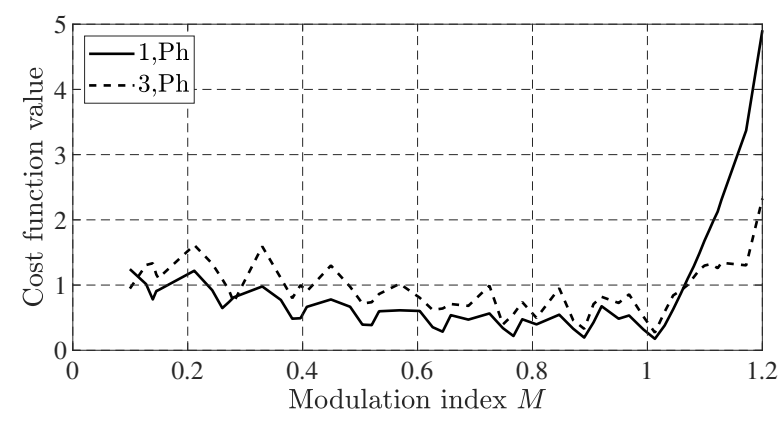

(a)

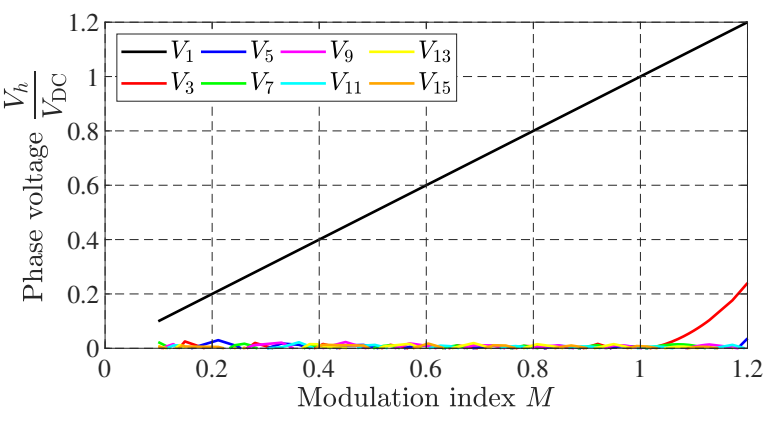

(b)

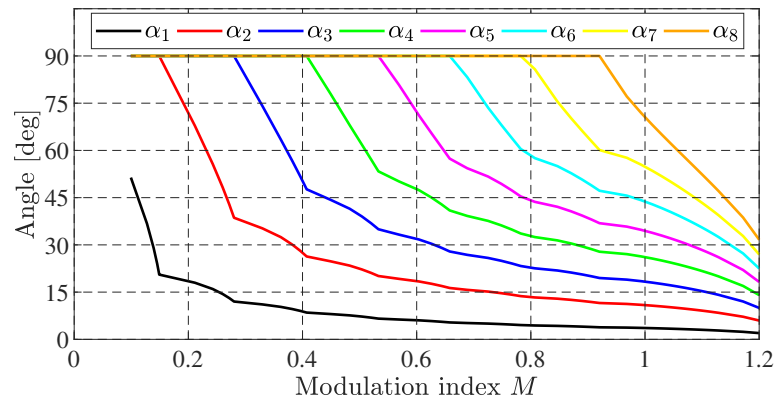

(c)

Fig. 4: (a) Cost function value, (b) harmonic components and (c) insertion angles relative to the modulation index $M$ when using NLC.

lab's Genetic Algorithm (see Appendix Listing 2). Within the remainder of this article, the optimization solutions are referred to as $\mathrm{GA}_{1, \mathrm{Ph}}$ and $\mathrm{GA}_{3, \mathrm{Ph}}$. The quantities $W T H D_{1, \mathrm{Ph}}$ and $W T H D_{3, \mathrm{Ph}}$ are used as benchmark parameters to asses the probable current quality.

Figure 3 (a) shows the sinusoidal reference voltage for a modulation index $M$ of 0.95 and the 


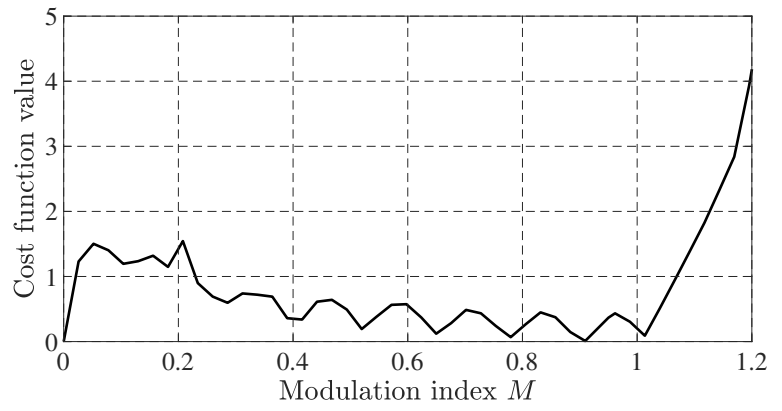

(a)

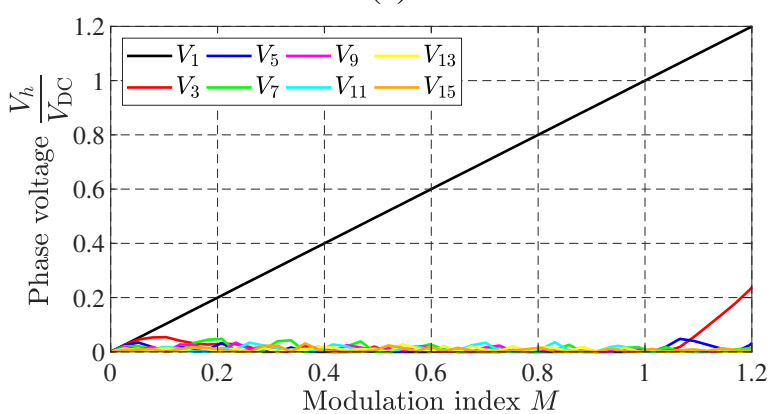

(b)

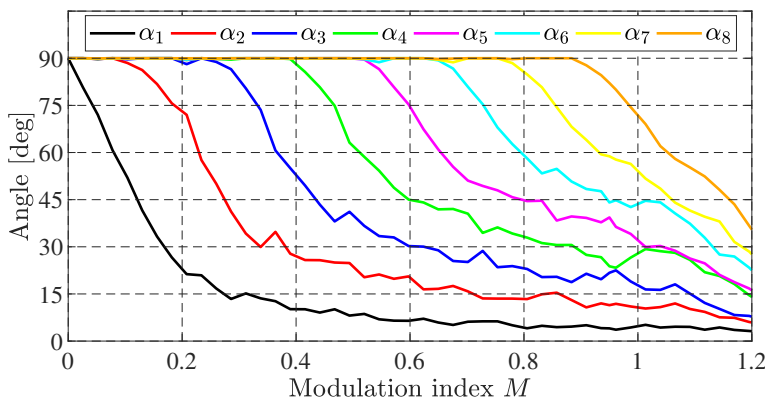

(c)

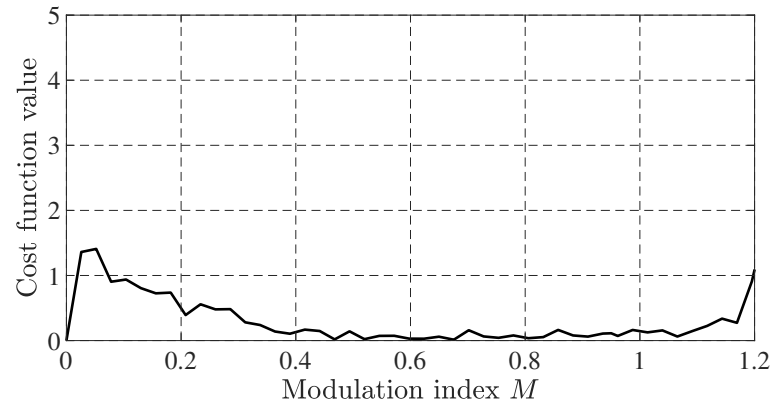

(d)

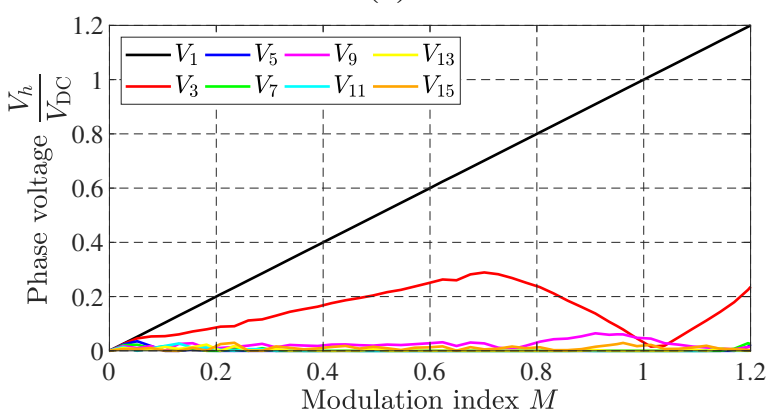

(e)

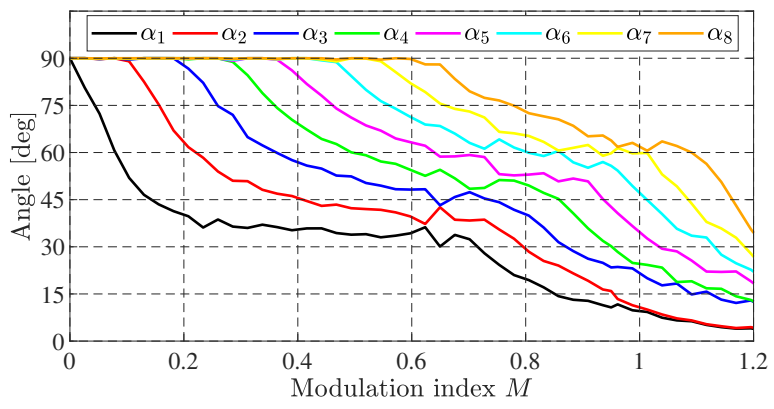

(f)

Fig. 5: (a) Cost function value, (b) harmonic components and (c) insertion angles relative to the modulation index $M$ when using the genetic algorithm optimization solution with respect to a series of odd harmonics (with optional grounding/single-phase). (d) Cost function value, (e) harmonic components and (f) insertion angles relative to the modulation index $M$ when using the genetic algorithm optimization solution with respect to a series of odd harmonics excluding the triplen harmonics (without optional grounding/three-phase).

corresponding staircase shaped output voltage waveforms. The blue waveform is synthesized using nearest-level control. The red and the green waveform are obtained using the optimized insertion angles when considering a single phase and a three-phase system without the optional grounding (see Fig. 1), respectively. The corresponding harmonic components of the waveforms are shown in Fig. 3 (b). As can be seen, the $\mathrm{GA}_{1, \mathrm{Ph}}$ solution reduces the magnitude of the low-order harmonics up to the $9^{\text {th }}$ order, whereas some of the higher order harmonics $\left(11^{\text {th }}, 17^{\text {th }}, 21^{\text {st }}, 23^{\text {rd }} \ldots\right)$ are increased in comparison to NLC. The value of the weighted THD, $W T H D_{1, \mathrm{Ph}}$, is marginally decreased when using the $\mathrm{GA}_{1, \mathrm{Ph}}$ solution in comparison to NLC. Nonetheless, considering the $\mathrm{GA}_{3, \mathrm{Ph}}$ solution, all of the targeted low-order harmonics are almost eliminated, whereas the triplen, low-order harmonics $\left(3^{\text {rd }}\right.$ and $\left.9^{\text {th }}\right)$ are increased. Therefore, the value of the $W T H D_{3, \mathrm{Ph}}$ is reduced from $0.25 \%$ to $0.09 \%$ when using the $\mathrm{GA}_{3, \mathrm{Ph}}$ solution in comparison to NLC.

The cost function value, the magnitude of the low-order harmonics components and the insertion angles relative to the modulation index for NLC and the genetic algorithm optimizations $\mathrm{GA}_{1, \mathrm{Ph}}$ and $\mathrm{GA}_{3, \mathrm{Ph}}$ can be seen in Figs. 4 and 5, respectively. The calculated values of the $W T H D_{1, \mathrm{Ph}}$ and $W T H D_{3, \mathrm{Ph}}$ 
relative to the modulation index $M$ for NLC in comparison to the optimization solutions $\mathrm{GA}_{1, \mathrm{Ph}}$ and $\mathrm{GA}_{3, \mathrm{Ph}}$ can be seen in Fig. 6. The modulation index from 0.5 to 1.0 is highlighted in both Fig. 6(a) and Fig. 6(b). On the one hand, similar as seen from Fig. 3, the optimization solution $\mathrm{GA}_{1, \mathrm{Ph}}$ does not significantly improve the $W T H D_{1, \mathrm{Ph}}$. Thus, an optimization of the insertion angles for a single phase system or a three-phase system with both star points grounded does not seem reasonable, since NLC is achieving a similar current quality according to the obtained $W T H D_{1, \mathrm{Ph}}$ values. On the other hand, when considering a three-phase system without the additional grounding, the value of the $W T H D_{3, \mathrm{Ph}}$ can be reduced when using the $\mathrm{GA}_{3, \mathrm{Ph}}$ solution in comparison to NLC, as shown in Fig. 6(b). As can be seen, for certain values of the modulation index $M$, the $W T H D_{3, \mathrm{Ph}}$ can be reduced by a factor of about 3 to 4, whereas for some other values of the modulation index $M$ the improvement is almost negligible. However, since the optimized $\mathrm{GA}_{3, \mathrm{Ph}}$ solution does not necessarily contain the global optimum insertion angles, the presented solution could be enhanced further.

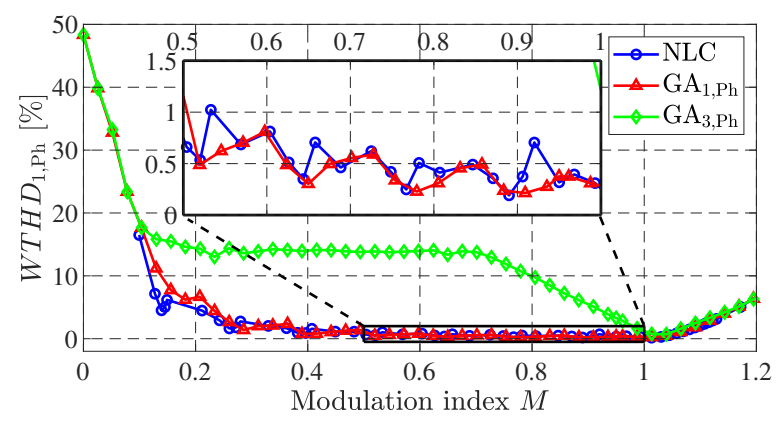

(a)

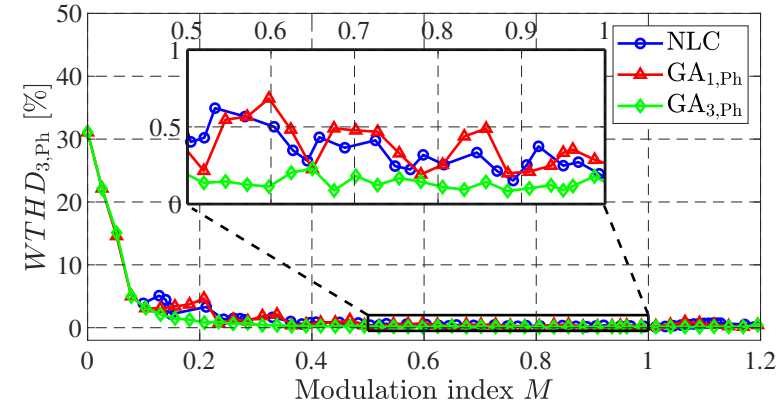

(b)

Fig. 6: Obtained values of the (a) $W T H D_{1, \mathrm{Ph}}$ and (b) $W T H D_{3, \mathrm{Ph}}$ relative to the modulation index $M$ for NLC in comparison to the optimization solutions $\mathrm{GA}_{1, \mathrm{Ph}}$ and $\mathrm{GA}_{3, \mathrm{Ph}}$.

\section{Conclusion}

This paper has dealt with an elimination/mitigation technique of a selection of a multilevel converter's low-order output voltage harmonics when using fundamental frequency switching. In comparison to nearest-level control, its effect, when operating in a single or three-phase arrangement (without both star points grouned), has been assessed using the concept of the weighted total harmonic distortion, WTHD.

Using fundamental frequency switching, an $L$-level multilevel converter, when operating at a high modulation index $M$, can control up to $\frac{L-1}{2}$ output voltage components, including the fundamental component. To achieve a proper elimination, the insertion angles $\alpha$ must be properly determined in an offline calculation or optimization, requiring a high computational effort. It has been shown that the nonlinear set of expressions of the output harmonics can be linearized using Chebyshev polynomials. Thus, solving the set of linearized equations numerically seems convenient for up to nine output voltage levels. For more than nine output voltage levels, the computation time increases significantly. Therefore, when having more than nine output voltage levels, it has been suggested to use an optimization approach instead, utilizing for example Matlab's genetic algorithm. Nonetheless, a sweep of optimizations relative to the modulation index has showed that it is quite difficult to find the proper insertion angles to eliminate all selected harmonic components completely.

Nevertheless, from the obtained results for a suggested 17-level converter, the following conclusions can be drawn: When operating in a three-phase arrangement (without the additional grounding), the current quality can be significantly increased when using an optimized output voltage waveform. A wide selection of low-order harmonics are reduced, whereas the triplen harmonics are increased, but these do not create any currents. However, when operating in a single-phase arrangement, the current quality cannot be improved when using an optimized voltage waveform. 


\section{Appendix}

Listing 1: Numerical solution using trigonometric identities and Matlab command vpasolve().

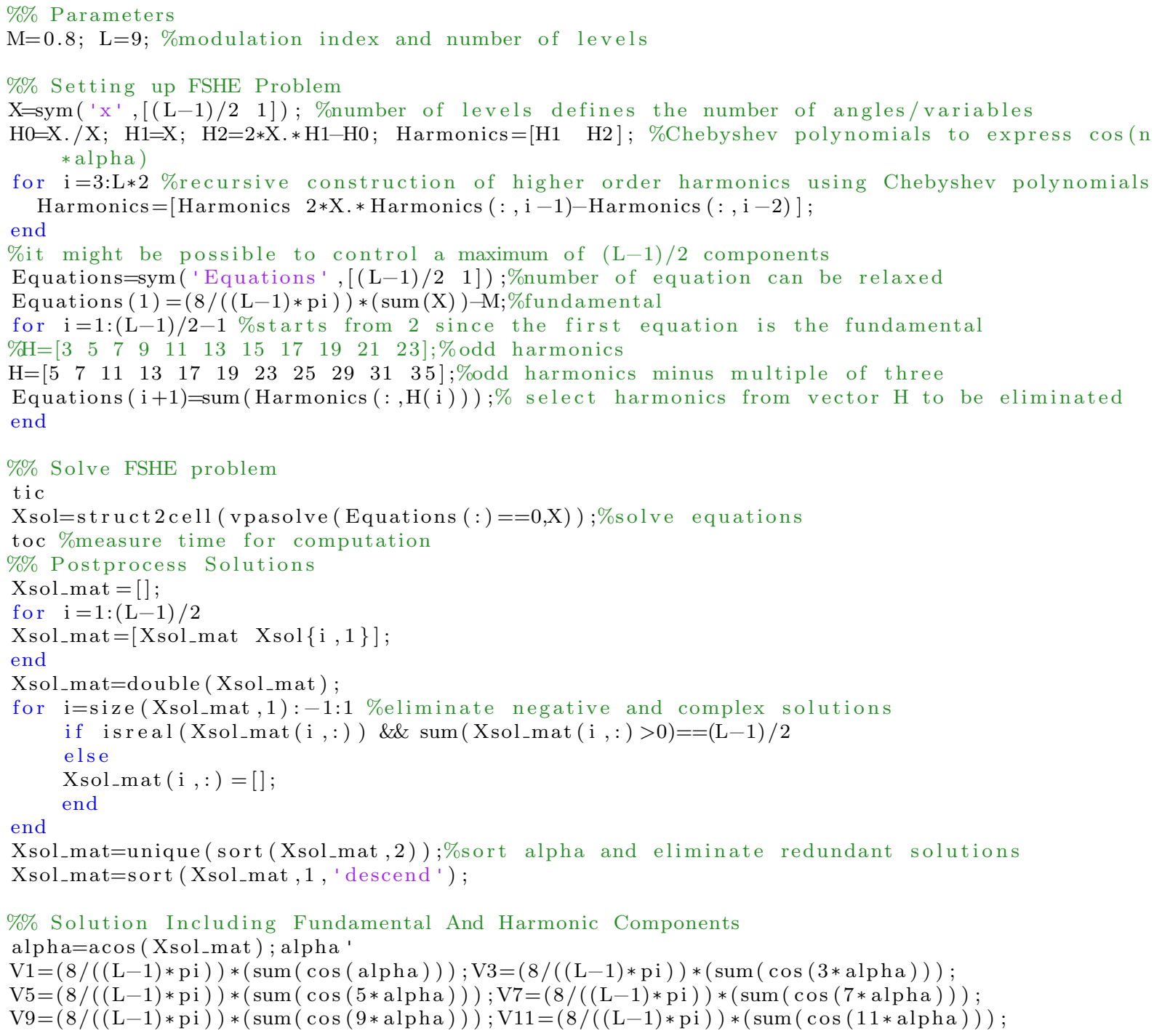

Using a computer with an intel i7-7700 and $64 \mathrm{~Gb}$ of RAM, the computation time of the above code for a nine-level output waveform with a modulation index of 0.8 was about $7 \mathrm{~min}$ and $18 \mathrm{~s}$. The obtained result can be seen below:

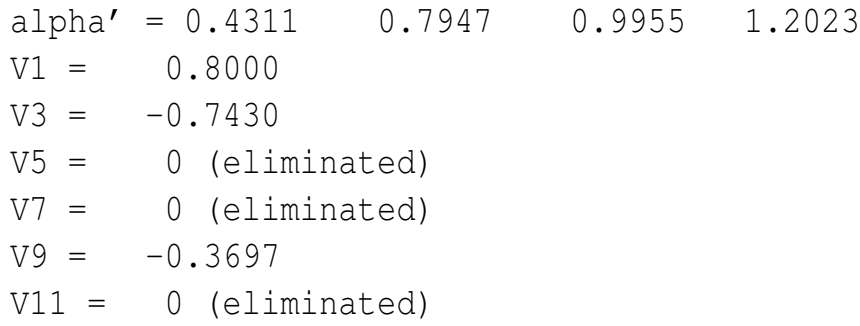

Listing 2: Optimization using Matlab's genetic algorithm.

$\%$ Parameters

M_sweep=linspace $(0.0,4 / \mathrm{pi}, 50) ; \mathrm{L}=17 ; \%$ modulation index sweep and number of levels M_sweep $=\left[\begin{array}{ll}0.8 & 0.95\end{array}\right]$

$\%$ Setting up FSHE Problem nvars $=(\mathrm{L}-1) / 2$; \%umber of levels defines the number of angles/variables $\mathrm{A}=\mathrm{zeros}($ nvars +1, nvars $)$; 


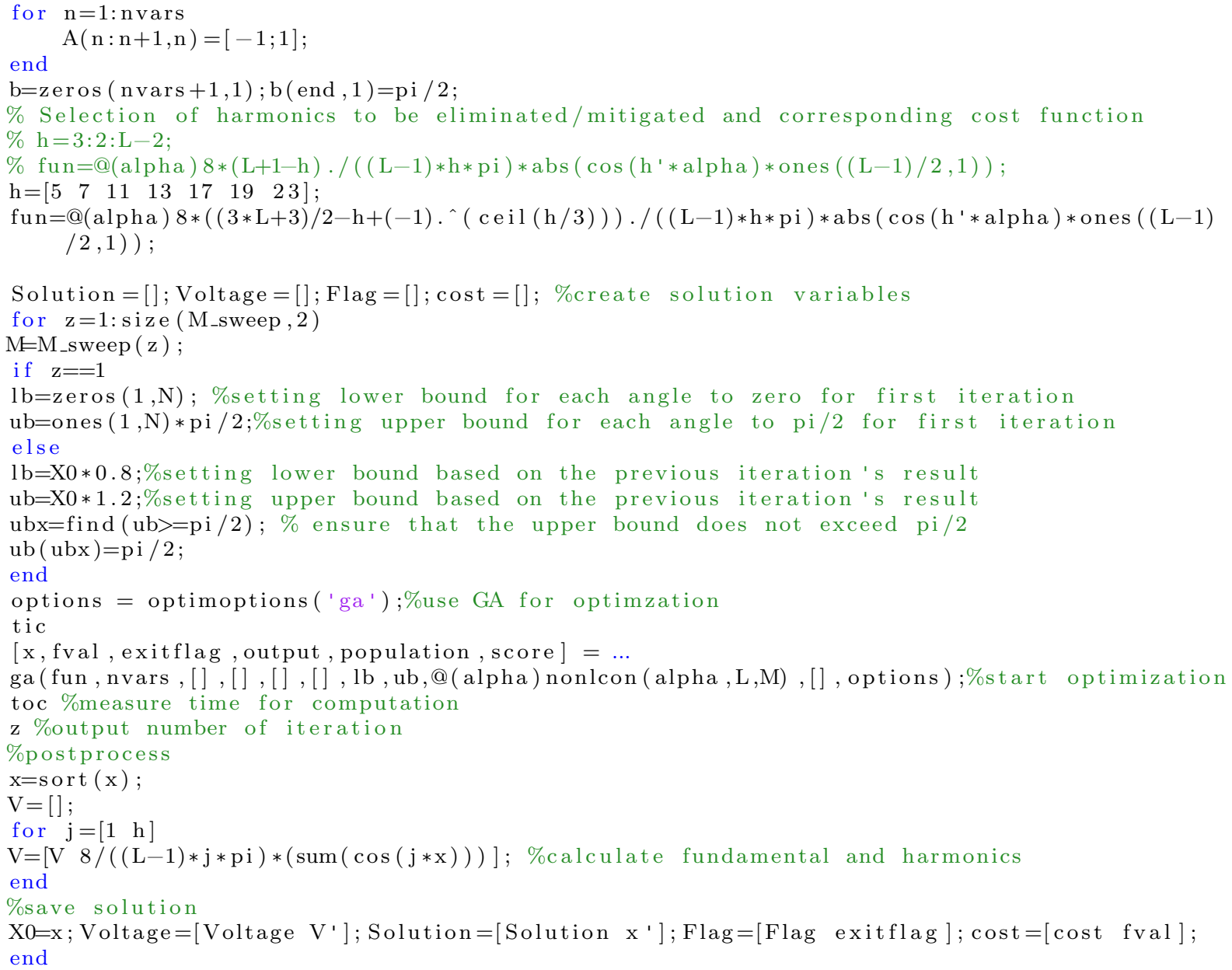

\section{References}

[1] A. Lesnicar and R. Marquardt, "An innovative modular multilevel converter topology suitable for a wide power range," in 2003 IEEE Bologna Power Tech Conference Proceedings, vol. 3, 2003, pp. 6 pp. Vol.3-.

[2] L. M. Tolbert, Fang Zheng Peng, and T. G. Habetler, "Multilevel converters for large electric drives," IEEE Transactions on Industry Applications, vol. 35, no. 1, pp. 36-44, 1999.

[3] K. Corzine and Y. Familiant, "A new cascaded multilevel h-bridge drive," IEEE Transactions on Power Electronics, vol. 17, no. 1, pp. 125-131, 2002.

[4] O. Josefsson, A. Lindskog, S. Lundmark, and T. Thiringer, "Assessment of a multilevel converter for a phev charge and traction application," in The XIX International Conference on Electrical Machines - ICEM 2010, 2010, pp. 1-6.

[5] L. E. M. Calaça, E. G. A. Jesus, and J. Dionisio Barros, "Multilevel converter system for photovoltaic panels," in 2016 IEEE International Conference on Renewable Energy Research and Applications (ICRERA), 2016, pp. 913-918.

[6] B. Alikhanzadeh, A. Bahmani, and T. Thiringer, "Efficiency investigation of 2l-dab and ml-dab for high-power pv applications," in 2018 Thirteenth International Conference on Ecological Vehicles and Renewable Energies (EVER), 2018, pp. 1-6.

[7] D. B. Cobaleda, M. Vivert, R. Diez, G. Perilla, D. Patiño, and F. Ruiz, "Low-voltage cascade multilevel inverter with gan devices for energy storage system," in 2019 IEEE 13th International Conference on Power Electronics and Drive Systems (PEDS), 2019, pp. 1-5.

[8] F. Helling, J. Glück, A. Singer, H.-J. Pfisterer, and T. Weyh, "The ac battery-a novel approach for integrating batteries into ac systems," International Journal of Electrical Power \& Energy Systems, vol. 104, pp. 150-158, 2019. 
[9] W. Han, T. Wik, A. Kersten, G. Dong, and C. Zou, "Next-Generation Battery Management Systems: Dynamic Reconfiguration," IEEE Ind. Electron. Mag., 2020, accepted.

[10] A. Kersten, K. Oberdieck, A. Bubert, M. Neubert, E. A. Grunditz, T. Thiringer, and R. W. De Doncker, "Fault detection and localization for limp home functionality of three-level npc inverters with connected neutral point for electric vehicles," IEEE Transactions on Transportation Electrification, vol. 5, no. 2, pp. 416-432, 2019.

[11] A. Kersten, E. Grunditz, and T. Thiringer, "Efficiency of active three-level and five-level npc inverters compared to a two-level inverter in a vehicle," in 2018 20th European Conference on Power Electronics and Applications (EPE'18 ECCE Europe), 2018, pp. P.1-P.9.

[12] A. Kersten, M. Kuder, E. Grunditz, Z. Geng, E. Wikner, T. Thiringer, T. Weyh, and R. Eckerle, "Inverter and battery drive cycle efficiency comparisons of chb and mmsp traction inverters for electric vehicles," in 201921 st European Conference on Power Electronics and Applications (EPE '19 ECCE Europe), 2019, pp. P.1-P.12.

[13] A. Acquaviva, A. Rodionov, A. Kersten, T. Thiringer, and Y. Liu, "Analytical conduction loss calculation of a mosfet three-phase inverter accounting for the reverse conduction and the blanking time," IEEE Transactions on Industrial Electronics, pp. 1-1, 2020.

[14] F. Chang, O. Ilina, M. Lienkamp, and L. Voss, "Improving the overall efficiency of automotive inverters using a multilevel converter composed of low voltage si mosfets," IEEE Transactions on Power Electronics, vol. 34, no. 4, pp. 3586-3602, 2019.

[15] A. Kersten, K. Oberdieck, J. Gossmann, A. Bubert, R. Loewenherz, M. Neubert, E. Grunditz, T. Thiringer, and R. W. De Doncker, "Cm line-dm noise separation for three-level npc inverter with connected neutral point for vehicle traction applications," in 2019 IEEE Transportation Electrification Conference and Expo (ITEC), 2019, pp. 1-6.

[16] A. Kersten, K. Oberdieck, J. Gossmann, A. Bubert, R. Loewenherz, M. Neubert, T. Thiringer, and R. De Doncker, "Measuring and separating conducted three-wire emissions from a fault-tolerant, npc propulsion inverter with a split-battery using hardware separators based on hf transformers," IEEE Transactions on Power Electronics, pp. 1-1, 2020.

[17] Y. Yang, H. Wen, M. Fan, M. Xie, S. Peng, M. Norambuena, and J. Rodriguez, "Computationefficient model predictive control with common mode voltage elimination for five-level anpc converters," IEEE Transactions on Transportation Electrification, pp. 1-1, 2020.

[18] D. G. Holmes and T. A. Lipo, Pulse width modulation for power converters: principles and practice. John Wiley \& Sons, 2003, vol. 18.

[19] K. Sharifabadi, L. Harnefors, H.-P. Nee, S. Norrga, and R. Teodorescu, Design, control, and application of modular multilevel converters for HVDC transmission systems. John Wiley \& Sons, 2016.

[20] J. N. Chiasson, L. M. Tolbert, K. J. McKenzie, and Zhong Du, "Elimination of harmonics in a multilevel converter using the theory of symmetric polynomials and resultants," IEEE Transactions on Control Systems Technology, vol. 13, no. 2, pp. 216-223, 2005.

[21] J. Chiasson, L. M. Tolbert, K. McKenzie, and Zhong Du, "A complete solution to the harmonic elimination problem," in Eighteenth Annual IEEE Applied Power Electronics Conference and Exposition, 2003. APEC '03., vol. 1, 2003, pp. 596-602 vol.1.

[22] J. N. Chiasson, L. M. Tolbert, K. J. McKenzie, and Zhong Du, "Control of a multilevel converter using resultant theory," IEEE Transactions on Control Systems Technology, vol. 11, no. 3, pp. 345354, 2003.

[23] B. Ozpineci, L. M. Tolbert, and J. N. Chiasson, "Harmonic optimization of multilevel converters using genetic algorithms," in 2004 IEEE 35th Annual Power Electronics Specialists Conference (IEEE Cat. No.04CH37551), vol. 5, 2004, pp. 3911-3916 Vol.5.

[24] M. Kuder, A. Kersten, L. Bergmann, R. Eckerle, F. Helling, and T. Weyh, "Exponential modular multilevel converter for low voltage applications," in 201921 st European Conference on Power Electronics and Applications (EPE'19 ECCE Europe), 2019, pp. P.1-P.11. 